Ken Farø*

\title{
Können geflügelte Worte "wissenschaftlich" sein, oder gehören sie ins Raritätenkabinett? Eine Diskussion anlässlich zweier Neuerscheinungen ${ }^{1}$
}

\begin{abstract}
How are we to understand and define stock quotations (Germ. geflügelte Worte, Dan. bevingede ord)? This is in my view far too important a task to leave to the publications collecting a highly interesting but nevertheless often heterogenous material under the header of this term. The material published does, so it seems, often not meet the requirements of this linguistic category when systematically defined. Two basic criteria must in my view be fulfilled if we are to speak of stock quotations: (1) the speech act citation (in broad terms) must be a genuine feature of the linguistic unit, and (2) the lexeme must be lexicalized. Other features such as polylexical structure and modification may be optional but they should, I suggest, not be seen as obligatory. Stock quotations are both a lexical as well as a highly pragmatic category as their genuine purpose is to perform a specific speech act. The discussion of stock quotations is undertaken on the grounds of two new dictionaries of this category, i.e. the German Büchmann (2002) and the Danish Bramsen (2001). Stock quotations do - in spite of what is claimed in one of these dictionaries - in fact lend themselves to scientific description.
\end{abstract}

\section{1. $\quad$ Einleitung}

Dem Vorwort einer (relativ) neuen dänischen Sammlung geflügelter Worte zufolge (= Bramsen 2001: 11) "[sind] geflügelte Worte [...] keine Wissenschaft und können es auch nicht sein" (meine Übers.) ${ }^{2}$. Obwohl damit sicherlich nicht gemeint ist, dass geflügelte Worte $(\mathrm{gW})$ an sich nicht wissenschaftlich sind -

\footnotetext{
1 (1) Joachim Bo Bramsen/Bo Bramsen (2001): Bevingede ord og aforismer. En citatordbog. Kopenhagen: Politiken. 495 S., 249 dkr. (2) Georg Büchmann et al. (2002): Der neue Büchmann. Geflügelte Worte. München: Bassermann. 695 S., 10 Euro.

2 Dänisch: "Bevingede ord er ingen videnskab og kan ikke være det".

* Universität Kopenhagen

Institut für Englisch, Germanisch und Romanisch

Njalsgade 120

DK-2300 Kopenhagen $S$
}

Hermes, Journal of Linguistics no 35-2005 
auf welche zu beschreibenden Objekte würde dies schon zutreffen? ${ }^{3}-$, ist dies eine Behauptung, die jeden Wissenschaftler provozieren sollte. Denn sollte es sich hier tatsächlich um ein Gebiet handeln, das sich wissenschaftlicher Beschreibung entzieht? Urban (in: Büchmann 2002: 7) geht in die gleiche Richtung, wenn er schreibt, dass "eine letztgültige Objektivität nicht zu erreichen" sei.

Die Skepsis wissenschaftlicher Systematik gegenüber, die diese Zitate vermitteln, bilden einen passenden Eingang zu diesem Beitrag. Denn er unternimmt - in einer expliziten Gegenposition zur besprochenen Haltung - den Versuch, tatsächlich einer wissenschaftlichen Beschäftigung mit den $\mathrm{gW}$ den Weg zu ebnen, indem er ein systematisches Konzept vorschlägt. Denn ein solches steht $\mathrm{m}$. E. noch aus.

Ziel des Beitrags ist eine Analyse und Diskussion des theoretischen und praktischen Umgangs mit $\mathrm{gW}$, hauptsächlich im Deutschen und Dänischen. Dabei wird sowohl auf Linguisten- als auch auf Laiengesichtspunkte eingegangen, denn die Bezeichnung geflügeltes Wort (dänisch: "bevinget ord"4) ist wohl den meisten Sprechern beider Sprachgebiete bekannt, was nicht zuletzt für die Lexikografen eine relevante Tatsache ist. Denn was macht man, wenn die potenziellen Benutzer dieser Nachschlagewerke tatsächlich keine einheitliche Vorstellung davon haben, was $\mathrm{gW}$ sind? Soll man etwa dann versuchen, so viele Auffassungen wie möglich zu befriedigen, oder soll man dieses Problem einfach außer Acht lassen? Alles hängt von der Definition und deren Einhaltung ab, auch in diesem Fall. Die Frage ist, wie gW definiert werden sollen und welche lexikalischen Einheiten von der lexikografischen Praxis darunter subsummiert werden?

\section{Das Problem}

Geflügelte Worte sind eine lexikalische Kategorie, die bisher von der systematischen Forschung weithin vernachlässigt wurde. Dass es sich zunächst um eine genuine Wortschatzeinheit handelt, steht $\mathrm{m}$. E. außer Frage. Die Zurückhaltung der Forschung auf diesem Gebiet mag damit zusammenhängen, dass $\mathrm{gW}$ mit einigem Recht als eine sehr heterogene Kategorie angesehen wird, und

\footnotetext{
${ }^{3}$ Das Ohr ist z. B. an sich keine Wissenschaft, lässt sich aber durchaus wissenschaftlich beschreiben. Es handelt sich um die bekannte Differenzierung zwischen einer Objektund einer Metaebene. Alles, was an sich intuitiv "unwissenschaftlich" erscheinen mag, darunter sogar die Astrologie, lässt sich auch zumindest wissenschaftlich beschreiben, wenn auch nicht immer betreiben. Lexikografie etwa kann bekanntlich sowohl wissenschaftlich beschrieben als auch betrieben werden.

${ }^{4}$ Die dänische Bezeichnung ist eine genaue Trajektion des büchmannschen Terminus.
} 
dass der Kern der $\mathrm{gW}$ vielleicht nicht im engeren Sinn linguistisch ist, was in beiden Fällen einer Systematisierung Schwierigkeiten in den Weg legen dürfte. Die Frage ist aber, ob wir nach 140 Jahren - Büchmanns Wörterbuch erschien 1864 zum ersten Mal und führte gleichzeitig den Begriff $\mathrm{gW}$ in die Sprachwissenschaft ein - zu einem wirklichen Verständnis dieser Kategorie gekommen sind? Meine Behauptung ist, dass dies noch nicht der Fall ist. Im Gegenteil: Die Kategorie hat, so wie sie heute meistens gehandhabt und von vielen Linguisten und Laien verstanden wird, im Grunde genommen keine wissenschaftliche Legitimität. Das heißt aber nicht, dass die Bezeichnung $\mathrm{gW}$ dann auch keinen Sinn machen könnte, wenn man auf das fokussieren würde, was diese Kategorie tatsächlich leistet und was sie von anderen lexikalischen Kategorien unterscheidet. Dass die Linguistik eine Kategorie $\mathrm{gW}$ braucht, hoffe ich mit diesem Beitrag zu zeigen. Gleichzeitig soll aber demonstriert werden, wie problematisch der Begriff in der Praxis manchmal verwendet wird, und zwar anhand zweier neuer $\mathrm{gW}$-Wörterbücher, das eine deutsch (= Büchmann 2002), das andere dänisch (= Bramsen 2001).

\section{Was sind gW? - 3 Zugänge}

Um herauszufinden, was unter einem $\mathrm{gW}$ zu verstehen ist, kann man die Frage auf verschiedene Weise stellen, z. B. 1) als Frage an Laien und Semilinguisten, d. h. wie wird er im allgemeinen und semifachlichen Sprachgebrauch verstanden?; 2) als Frage an Linguisten; 3) schließlich kann man aus der lexikografischen Praxis eine Auffassung induktiv herausdestillieren.

\section{1. "Die" Laienauffassung}

Fragt man (durchaus intelligente) Laien, was die Kategorie $\mathrm{gW}$ konstituiert, so bekommt man äußerst unterschiedliche Antworten. Z. B. handelt es sich um "kluge Worte", um "häufig verwendete Audrücke", es wird das Beispiel "Titel von bekannten Liedern" erwähnt, und es wird auf "bekannte Leute" hingewiesen ${ }^{5}$.

Eine andere kleinformatige Untersuchung unter angehenden dänischen Germanisten ${ }^{6}$ führte zu einem ähnlichen Ergebnis: Für den einen sind $\mathrm{gW}$ ein "etwas gehobener Sprachgebrauch", was ein anderer als "etwas gehoben" bezeichnet. Jemand hat darunter (zwar mit einem Fragezeichen versehen) sogar "ein Wort

\footnotetext{
${ }_{5}^{5}$ Ich habe eine Menge Personen meiner (nicht-linguistischen) Umgebung gefragt, und keiner hat die gleichen Vorstellungen davon gehabt. Würde man die Interview-Basis wesentlich erweitern, so wären wohl noch mehr Alternativen zu erwarten.

6 Im einem Seminar über Phraseologie im FS 03 an der Universität Kopenhagen.
} 
mit diversen Bedeutungen" assoziert. Nur ein einziger hat geantwortet: "ein Ausdruck aus einem klassischem Werk o. ä., der zu einer festen Wendung geworden ist". Viele haben die Frage erst gar nicht beantwortet. Doch wenn es um Beispiele geht, kommen m. E. durchaus adäquate Antworten:

(1) Heureka! (wird mit Archimedes assoziert)

(2) da steh' ich nun, ich armer Tor (aus Goethes Faust)

(3) das ist des Pudels Kern (do.)

Trotz dieser paar Einzelfälle muss wohl daran festgehalten werden, dass unter Nicht- und Semi-Linguisten von sehr heterogenen Vorstellungen vom Begriff $\mathrm{gW}$ auszugehen ist. Die Beispiele zeigen aber gleichzeitig, dass doch eine gewisse Einigkeit über Grundsätzliches zu bestehen scheint.

\section{2. "Die" linguistische Auffassung}

Angesichts dieser Heterogenität des gW-Begriffs in der Auffassung der Sprecher scheint es geraten, die Linguistik zu fragen, was im Grunde genommen die Kategorie der $\mathrm{gW}$ ausmacht. Wie äußern sich die gängigen Nachschlagewerke dazu? Zunächst ist festzustellen, dass die Kategorie in einigen Fällen von den Lexika gar nicht berücksichtigt wird. So ist sie z. B. in Bußmann (1989) nicht kodifiziert, was den Eindruck erstärkt, dass es sich um keine "wissenschaftliche" Kategorie handelt (vgl. Abschnitt 1.) Demgegenüber sind eine Menge an Nachschlagewerken zu verzeichnen, die sie erwähnen. Ich gehe hier bloß auf eine kleine Auswahl ein:

Glück (2000), der die Pluralform Geflügelte Worte wählt, verhält sich der Definition Robert-Tornows, dem Bearbeiter der ersten Ausgaben nach dem Tod Büchmanns, gegenüber loyal: "ein landläufiges Citat, [...] ein in weiteren Kreisen des Vaterlandes dauernd angeführter Ausspruch, Ausdruck oder Name, gleichviel welcher Sprache, dessen historischer Urheber, oder dessen literarischer Ursprung nachweisbar ist”. In Klosa et al. (2001) wird ein gW kurz und bündig als "bekannter, viel zitierter Ausspruch" definiert. Auch Burger (2003: 45) nimmt wie Glück auf Robert-Tornows Definition Bezug, nur modernisiert er dessen Konzeption, indem er explizit betont, dass heute ein rein literarischer Zugang überholt wäre. Somit müssen Bereiche wie Werbung und Film mit berücksichtigt werden, die wohl heute überhaupt zu den wichtigsten $\mathrm{gW}$-Spenderbereichen gehören. Schließlich lautet die Definition bei Becker-Christensen et al. (1999): "Ein bekanntes Literaturzitat, eine historische Äußerung, eine Redewendung, ein Schlagwort u. w. m., das auf eine bestimmte Quelle zurückgeführt werden kann” (meine Übers., $K F$ ).

Wenn man diese Ausführungen zusammenfasst, fällt zunächst die Einigkeit darüber auf, dass es sich beim $\mathrm{gW}$ offensichtlich um einen lexikalisierten "Aus- 
druck"7 handelt (= "bekannt", "landläufig"). Außerdem ist überall von einem Zitat die Rede, was uns hier besonders interessieren soll. Denn was ist unter einem "Zitat" zu verstehen? Diese Frage soll uns weiterhin beschäftigen.

\subsection{Die lexikografische Praxis}

\subsubsection{Einleitung}

Im Zentrum dieses Aufsatzes stehen zwei Neuerscheinungen zu gW, nämlich das deutsche Der neue Büchmann (= Büchmann 2002) und das dänische Bevingede Ord \& Aforismer (= Bramsen 2001). Man mag das Folgende zum Teil als eine Rezension dieser beiden Werke lesen, doch werde ich den Forderungen an die Wörterbuchrezension nicht Genüge leisten (vgl. Bergenholtz/Mogensen 1993), sondern die beiden Wörterbücher werden einzig unter dem Aspekt der Frage nach der Intension des Begriffes $\mathrm{gW}$ untersucht.

\subsubsection{Die Wörterbücher}

Das Werk, das sui generis mit der Kategorie $\mathrm{gW}$ verbunden wird, ist Büchmann (2002), obwohl sich m. E. darüber streiten lässt, ob tatsächlich der Name Büchmann "selber zum geflügelten Wort geworden ist" (op. cit.: 13) ${ }^{8}$. Seit seinem ersten Erscheinen sind mehrere Konkurrenten hinzugekommen (wie etwa Gerr 2001), doch hat "der" Büchmann seine Position als das Wörterbuch der gW bewahrt.

Zu seinem dänischen Pendant: Seit dem Erscheinen von T. Vogel-Jørgensens Bevingede ord ('Geflügelte Worte') im Jahr 1940 (heute: Vogel-Jørgensen 1998), ist dieses so gut wie ohne Konkurrenz gewesen. Seine bisher monopolhafte Stellung verdankte sich dieses Wörterbuch der Tatsache, dass es sich um eine gattungsmäßige Nische handelt, in die einzusteigen es sich für einen Verlag in einem kleinen Sprachgebiet wohl häufig nicht lohnt, weil der Aufbau eines Korpus von $\mathrm{gW}$ besonders große Mühe zu kosten scheint. Trotzdem ist im Verlag Politiken nun eine neue Publikation erschienen, die sich diese Kategorie zum Thema gemacht hat, nämlich Bramsen (2001). Der Verlag hatte übrigens schon 1958 eine gekürzte Fassung von Vogel-Jørgensen publiziert, aber seitdem habe Gad auf seine Rechte bestanden (Bramsen 2001: 10).

Im Folgenden werden die beiden neuen Wörterbücher auf ihren Inhalt hin

\footnotetext{
${ }^{7}$ Ich verwende hier diese vortheoretische Bezeichnung, weil ich mich an dieser Stelle nicht auf den strukturellen Charakter des gW's festlegen möchte (siehe unten).

${ }^{8}$ Es handelt sich hier eher, wie bei Kluge, Duden und Paul um eine Institutionalisierung des Namens.
} 
untersucht. Dabei werden die Lemmata in drei verschiedene Kategorien eingeteilt, nämlich in 1) diejenigen Formative, deren Status als $\mathrm{gW} \mathrm{m}$. E. indiskutabel ist; 2) eine Kategorie, die aus zweifelhaften $\mathrm{gW}$ besteht, und schließlich 3) solche Lemmata, die aus meiner Sicht keineswegs zu den $\mathrm{gW}$ gerechnet werden können.

\subsubsection{Genuine geflügelte Worte}

Zunächst einige Beispiele aus Büchmann (2002), die m. E. genuine geflügelte Worte sind:

(4) An der schönen blauen Donau (der Name eines Gesangwalzers von Johann Strauß)

(5) Soll ich meines Bruders Hüter sein? (laut der Bibel Kains Antwort an Gott, als er gefragt wird, wo sein Bruder Abel ist)

(6) Vom Winde verweht - (der deutsche Titel des amerikanischen Films Gone with the Wind)

(7) Angst essen Seele auf - (Titel eines Films von R.W. Fassbinder)

Aus Bramsen (2001) wären u. a. folgende Beispiele zu erwähnen:

(8) Var beredt ("sei(d) bereit" - Motto der dänischen Pfadfinder)

(9) Intet nyt fra Vestfronten ("Nichts Neues aus der Westfront" - die dänische Übersetzung von Im Westen nichts Neues)

(10) Det går ufatteligt godt ("es geht unglaublich gut" - der ehemalige konservative dänische Ministerpräsident Schlüter über die wirtschaftliche Lage des Landes)

Weitere Beispiele genuiner $\mathrm{gW}$, die aber in den beiden Wörterbüchern nicht enthalten sind, wären z. B.:

(11) Die Achse des Bösen - (George W. Bush)

(12) May the force be with you - (Darth Vader in "Star Wars")

(13) smagsdommer ("Geschmacksrichter" - Bezeichnung des dänischen Ministerpräsidenten Anders Fogh Rasmussen über angeblich unzumutbar moralisierende und politisierende staatsfinanzierte Forscher)

Bei all diesen Einheiten handelt es sich um Lexeme, die von den meisten Sprechern häufig als Zitate verwendet werden (s. u.) - die Beispiele 6, 7 und 12 sind übrigens gute Beispiele für die historische Erweiterung des Quellenbereichs, die Burger (1998: 41) erwähnt. Ausgangspunkt der Kategorisierung sollte dabei ein idealisierter 9 "Durchschnittssprecher" sein, weder der extraordinär ge-

9 Wie das durchaus in anderen linguistischen Zusammenhängen geschieht, vgl. etwa den Begriff idealer Sprecher/Hörer oder kompetenter Sprecher/Hörer. 
bildete noch der extrem bildungsarme Sprecher, der über keine oder geringe kulturell-historische Referenzen verfügt. Im ersteren Fall würden zu viele Lexeme als Zitate kategorisiert werden, weil der extrem gebildete Kompilator selbst über ein außerordentliches etymologisches und philologisches Wissen verfügt, so dass ihm die Quellen eines Phrasems oder eines Wortes oft bekannt sein werden. Im anderen Fall müsste man die Kategorie des gWs abschaffen, nämlich wenn der vollständig bildungsabstinente Bürger als Maßstab gelten sollte. Keine dieser beiden Alternativen wäre für eine adäquate Hantierung der Kategorie $\mathrm{gW} \mathrm{zu}$ empfehlen.

Zunächst ist aus diesen Einheiten ja aber keineswegs an sich einsehbar, dass sie Zitatcharakter haben. Dies ist nur anhand der konkreten sprachlichen Interaktion festzustellen. Als aufmerksamer Sprachbeobachter weiß man, dass gewisse Spracheinheiten dieses Merkmal tragen, andere nicht. Aber wie stellt man es konkret fest? Unten seien Beispiele von m. E. eindeutigen Zitat-Realisierungen der Einheiten angegeben:

(10a) Så når du ligesom vores statsminister siger: "det går ufatteligt godt", så er det ikke sikkert, at dine muskeltilhæftninger og ledbånd er klar til en øget belastning og mere træning (www.helse.dk)

(10b) Der skal nok komme søvnløse nætter og skrig og skrål, men den tid, den sorg. Det går ufatteligt godt, som Schlüter ville have sagt (www.supersarah.dk)

(10c) Som statsministeren engang udtrykte det; "det går ufatteligt godt". Blot kan vi sige det uden at feje noget ind under gulvtæppet (www.c-buro.dk)

(12a) May the force be with you. Dansk Star Wars Forening (dk.starwars.nu)

(12b) May the force be with you. Star Wars-fans begaves i disse tider (www.dron.dk)

(12c) Jeg vil bare sige at jeg elsker jeres musik, det er det fedeste jeg har hørt indtil videre af dansk rock! ... may the force be with you (www.gizmodesign.dk)

Was den Zitatcharakter direkt oder indirekt empirisch nachweisbar und damit intersubjektiv macht, sind verschiedene Faktoren: Manchmal wird das Zitieren explizit verbalisiert (10a-c). In anderen Fällen wird das Formativ außerhalb des Ursprungskontextes verwendet (alle Beispiele). Sonst ist auch bei (12a und b) klar, dass es sich um einen Hinweis auf den Ursprungskontext handelt. Im Fall (12c) ist dies nicht mehr zu entscheiden, aber die Existenz von vielen Beispielen wie (12a und b) legt die Interpretation nahe, dass auch mit (12c) zitiert, d. h. explizit auf den filmischen Kontext verwiesen wird. 
Das andere Kriterium, das erwähnt wurde, nämlich die Lexikalisierung, ist auch bei diesen Einheiten erfüllt. Man ist übrigens nicht länger dazu gezwungen, sich auf die eigene Introspektion zu verlassen, sondern Vermutungen lassen sich empirisch durch Korpora oder das Internet falsifizieren bzw. korrobieren. Dabei sollte immer darauf geachtet werden, dass es sich bei den Belegen tatsächlich um Zitate des Lexems handelt, und nicht um einfache Kopien des Ur-Textes oder Teile desselben (vgl. die obigen Beispiele). So erzielen die angeführten 2 Beispiele jeweils 268 und 3.430 hits im dänischen Teil von Google - wovon natürlich nicht alle Zitate sind -, was m. E. eindeutig auf Lexikalisierung hindeutet.

Diese beiden Kriterien sind zwar sehr unterschiedlich, aber trotzdem interdependent. Der Zitatcharakter muss jeweils in der einzelnen Äußerung nachgewiesen werden und die Zitat-Realisierungen müssen dann summativ einen so häufigen (und zerstreuten) Gebrauch ergeben, dass von Lexikalisierung gesprochen werden kann. Es reicht also nicht, dass eine Einheit einmal als Zitat nachgewiesen ist und insgesamt lexikalisiert zu sein scheint. Interessant sind in unserem Zusammenhang eigentlich nur die Zitat-Realisierungen. Jedes Wort oder Phrasem wird gelegentlich mal zitiert. Was zählt, ist aber nur das immer wieder Zitiertwerden. Dabei ist gerade dieses Kriterium am heikelsten, wie ich im nächsten Abschnitt zeigen möchte.

\subsubsection{Zum Merkmal "Zitathaftigkeit"}

Es wurde bereits vom Merkmal des Zitats gesprochen, das nicht nur von den Linguisten gefordert wird, sondern die gW-Lexikografen thematisieren den Begriff in ihren Nachschlagewerken auch. So ist bei Bramsen (2001) der Untertitel "Ein Zitatwörterbuch", während in Büchmann (2002) diese Bezeichnung im Anhang auftaucht, wo als systematischer Eingang in die Lemmaliste ein "Zitatenregister" abgedruckt ist. Der Begriff taucht auch in der Einleitung des Buches auf, aus der schon oben zitiert wurde.

Bisher habe ich den Begriff "Zitat" so benutzt, als wäre er recht unproblematisch. Einiges deutet aber darauf hin, dass gerade das "Zitat" keineswegs eine eindeutige Größe, sondern im Gegenteil ein ambiger Begriff ist, der in vielen Zusammenhängen benutzt wird, und der differenziert werden muss, wenn er als Teil-Kriterium von $\mathrm{gW}$ gelten soll. Z. B. werden nach Fleischer (1982: 80) Sprichwörter nicht wie lexikalische Einheiten "reproduziert", sondern eben "zitiert", was zwar als (Teil-)Kriterium zur Abgrenzung von Parömien gegenüber Idiomen und anderen Phrasemen gelten kann, die immer aktualisiert werden müssen, d. h. eventuelle Leerstellen und Wortformen müssen dem Kontext nach ausgefüllt werden bzw. flektiert werden. Trotzdem handelt es sich bei Sprichwörtern m. E. nicht um den Sprechakt eines Zitats. 
Dass eine solche Explizierung des Merkmals "Zitat" vonnöten ist, kann m. E. auch anhand folgender Beispiele aus den beiden Wörterbüchern gezeigt werden:

(14) tab og vind med samme sind ("verliert und gewinnt mit der gleichen Einstellung") (Motto des Kopenhagener Fußballvereins "Københavns Boldklub")

(15) som en hund ("wie ein Hund") (Kafka, Der Process)

(16) Die Blechtrommel (Roman von Günter Grass)

(17) Mehrwert (Karl Marx)

Diesen Lexemen fehlt m. E. die Zitathaftigkeit, und zwar aus recht verschiedenen Gründen: Bei (14) besteht eine rein zufällige Beziehung zwischen dem Motto und dem Klub. Im allgemeinen Sprachgebrauch wird der Fußballverein beim Gebrauch von tab og vind med samme sind keinesfalls zitiert, höchstens unter Mitgliedern des Klubs, und zwar aus dem Grund, dass es sich um ein allgemein verwendetes Sprichwort handelt, das vom Verein bloß aufgenommen worden ist. Hier vermittelt das Wörterbuch aber den falschen Eindruck einer inneren Zitat-Beziehung zwischen Klub und sprachlicher Einheit. Bei (15) hat der Autor offensichtlich ein zufälliges Idiom bei Kafka gefunden und es als gW abgedruckt. Dabei denkt kein Däne - und wohl auch kein Deutscher - an Kafka, wenn er dieses Idiom produziert oder rezipiert. Bei (16) ist das Problem eher, dass Die Blechtrommel zwar ein bekannter Titel ist, jedoch kaum zitiert, sondern bloß reproduziert werden kann. Wenn das Wort verwendet wird, dann wohl immer als direkte Bezugnahme auf das literarische Denotat, und eben nicht als Zitat. Das macht es $\mathrm{m}$. E. nicht zum gW, genauso wenig wie Dänemark geflügelt ist, weil es nicht zitiert werden kann. Und (15) ist ein Fachterminus, der auch kaum zitiert wird, auch wenn einige Ökonomen und Historiker seinen Ursprung kennen.

Das Zitat hat in der Sprachwissenschaft m. E. kaum einen terminologischen Status, sondern es wird meistens einfach als eindeutig vorausgesetzt. Das sieht man auch daran, dass renommierte und umfassende linguistische Lexika auf diesen Begriff gar nicht eingehen (vgl. Bußmann 1989 und Glück 2000). Ein Autor, der bei $\mathrm{gW}$ das Zitat-Merkmal immerhin spezifiziert, ist Burger (2003: 45). Er präzisiert den Begriff dahin, dass es entscheidend sei "daß bei den Sprechern ein Bewußtsein dafür vorhanden ist, daß der Ausdruck auf eine bestimmte und allenfalls bestimmbare Quelle zurückgeht”. Damit nähert er sich einer adäquaten Definition von "Zitat". Burger trifft $\mathrm{m}$. E. aber noch nicht den Kern des Problems. Denn wenn die Quelle nur "bestimmbar" ist, dann ist nicht völlig klar, ob es sich um eine eher philologische Kategorie handelt - was m. E. wissenschaftlich unattraktiv wäre -, oder ob Burger hier bereits den ersten 
Schritt hin zu einem genuin pragmatischen gW-Begriff getan hat, was aus meiner Sicht unbedingt zu empfehlen wäre.

Es handelt sich bei den $\mathrm{gW}$ um eine völlig andere Art des Ziterens als etwa bei Sprichwörtern und anderen lexikalisierten Komplexen, die einfach reproduziert werden. Der Gebrauch von $\mathrm{gW}$ als $\mathrm{gW}$ ist nämlich ein besonderer Sprechakt, der darin besteht, dass beim Gebrauch des Lexems bewusst zitiert wird, d. h. dass auf eine externe Textwelt (im weitesten Sinn) Bezug genommen wird. Es handelt sich somit keineswegs immer darum, dass man zu jeder Zeit weiß, wer zuerst das Lexem verwendet hat. In manchen Fällen ist das eigentlich irrelevant. Sondern interessant ist oft nur, mit welchem prominenten Sprecher, welchen äußeren Umständen etc. das Zitat (intendiert) in Verbindung gebracht wird, ohne dass es sich dabei um den Originaläußerer oder -Situation handeln muss. Hauptsache ist vor allem die Intention des Zitierers oder das Erlebnis des Hörers.

Man kann auch sagen, dass mit $\mathrm{gW}$ explizit auf Enzyklopädisches referiert wird, denn $\mathrm{gW}$ sind, obwohl sie sprachliche Einheiten sind, genuin extralinguistische Einheiten. Sie stehen, wenn sie wirkliche gW sind, mit der Außenwelt, sei es Geschichte, Literatur, Film, Politik o. a. m., in enger Verbindung.

Bei anderen lexikalischen Einheiten wird dem Sprachbenutzer und -Interpreten zwar auch gewissermaßen Einblicke in Außersprachliches wie etwa Kulturgeschichte gewährt (z. B. bei gewissen Idiomen und Sprichwörtern). Der für das Verständnis dieses Problems ganz entscheidende Unterschied ist aber, dass dies implizit und vom Sprecher nicht-intendiert geschieht. Das Zitieren im Falle des gWs ist also als ein pragmatischer Akt des Sprechers zu verstehen, der deshalb vielleicht auch als „explizites enzyklopädisches Referieren“ zu beschreiben wäre.

Wenn man anerkennt, dass das $\mathrm{gW}$ eine pragmatische, und keine philologische Kategorie ist, wird man auch akzeptieren müssen, dass es nach dieser Konzeption keineswegs ein Zeichen von "Geflügeltheit" sein kann, dass jemand "vielleicht" (Scholze-Stubenrecht et al. 1993: passim) der Urheber sein könnte, oder "als wahrscheinlichste Quelle" (ebd.: passim) angesehen werden könne, denn gerade das "Vielleicht" zeigt, dass es sich um kein Zitat im pragmatischen Sinn handelt - Zitat als Sprechakt - sondern um den Rekonstruktionsversuch einer Etymologie.

\subsubsection{Lexikalisierung}

Obwohl das Merkmal (pragmatisches) Zitat wichtig ist, muss m. E. die Devise gelten: Kein gW ohne Lexikalisierung. Denn viele Wörter oder Phraseme können einmal oder gelegentlich zitiert werden, ohne dass sie damit $\mathrm{gW}$ wären. Wenn wir am Merkmal der Lexikalisierung festhalten, so muss gefolgert wer- 
den, dass $\mathrm{gW}$-Kandidaten, die in sehr großen Textdatenbanken oder im Internet nicht oder fast nicht als Zitate vorkommen, kaum als geflügelt gelten können. In solchen Fällen handelt es sich wohl eher um idiolektale Präferenzen des Interpreten oder zufällig gefundene Bonmots, die aber mit den Gewohnheiten der Sprachmeinschaft nichts gemein hat. Gerade dies könnte bei den folgenden Beispielen der Fall gewesen sein, bei denen von einem mehr oder weniger fragwürdigen Grad an Lexikalisierung des Syntagmas gesprochen werden muss:

(18) Ein Kanadier, der noch Europens übertünchte Höflichkeit nicht kannte (Seume)

(19) Ha, Frau, das ist wider die Abrede (Lessing: Emilia Galotti)

(20) Auf die Postille gebückt, zur Seite des wärmenden Ofens (Voß)

(21) Men Clara! Hvorfor rødmer du? (Heiberg)

(22) Musikken udtrykker det, der ikke kan siges, og det, som det er umuligt at tie om (Victor Hugo)

(23) Bliv ikke for utilpas og skræmt over dine handlinger. Hele livet er en oplevelse (Ralph W. Emerson)

In großen Korpora oder in Suchmaschinen im Internet kommen sie nicht vor. Beide Wörterbücher sind voll solcher Beispiele, die m. E. wegen mangelnder Lexikalisierung aus dem Rahmen der $\mathrm{gW}$ fallen. Wohl bis zu einem Drittel aller Lemmata beider Wörterbücher leiten unter dieser Mangel.

\subsubsection{Weitere Merkmale?}

Werden $\mathrm{gW}$ überhaupt linguistisch behandelt, dann meistens in einem phraseologischen Zusammenhang. Dies ist pragmatisch (jetzt im gemeinsprachlichen Sinn zu verstehen) durchaus vertretbar (Farø 2003), jedoch kaum systematisch adäquat. Wörter wie:

(24) Wirtschaftswunder (Ludwig Erhard)

(25) Lorteland! ('Scheißland', Buster Larsen, dän. Kabarettist)

(26) smagsdommer ('Geschmacksrichter'; dän. Ministerpräsident Anders Fogh Rasmussen)

(27) Glasnost und Perestrojka (Gorbatschow)

(28) Heureka! (Archimedes)

(29) papirnusser (etwa 'Papierkrämer', für Beamte etc.; Mogens Glistrup, dän. Rechtspolitiker)

sind nämlich auch geflügelt, weil sie häufig mit Zitatintention verwendet oder als Zitate empfunden werden. Es kann m. E. deshalb ausgeschlossen werden, dass Polylexikalität ein notwendiges Kriterium für $\mathrm{gW}$ ist, auch wenn sie sehr 
oft mit diesem Merkmal kovariieren. GW sind deshalb keine echte Teilmenge der Phraseologie.

Nicht selten werden $\mathrm{gW}$ im Sprachgebrauch modifiziert. Unten seien einige Beispiele für dieses Phänomen angegeben:

(30) Jeg tænker, altså er jeg ikke James Brown ('ich denke, also bin ich nicht James Brown') (www.afsnitp.dk; - zitiert Descartes: cogito ergo sum)

(31) Ein Lied geht durch Europa (www.existenzgeld.de; - zitiert Karl Marx: Ein Gespenst geht durch Europa)

(32) I Danmark er jeg født - men hvor har jeg hjemme? ('In Dänemark bin ich geboren - aber wo ist mein Zuhause?) (www.dm-dk.dk; zitiert Hans Christian Andersens Lied: I Danmark er jeg født, der har jeg hjemme 'dort ist mein Zuhause')

(33) Völker, schaut auf dieses Dach! (www.erneuerbareenergien.de; zitiert Ernst Reuter (Oberbürgermeister Berlins im Kalten Krieg): Völker schaut auf diese Stadt!)

Einige Forscher meinen (pers. Mitt.), Modifikation sollte ein notwendiges Kriterium von $\mathrm{gW}$ sein. Ich gebe zu, dass Modifikationen ein gutes Indiz für $\mathrm{gW}$ sein können. Es kann m. E. jedoch weder ein notwendiges noch ein hinreichendes Kriterium für $\mathrm{gW}$ sein. Viele $\mathrm{gW}$ werden niemals modifiziert, während Idiome häufig modifiziert werden, ohne das Merkmal Zitat zu tragen, weshalb sie aus meiner Sicht keine $\mathrm{gW}$ sein können.

\subsubsection{Wenn die Flügel zu weit tragen: Beispiele, die die Kategorie gW sprengen}

Um zu zeigen, wie weit sich die gW-Konzeption von den genuinen $\mathrm{gW}$ entfernen kann, wenn ihr keine operationalisierbare Definition zugrunde liegt, möchte ich ein paar extreme Beispiele zeigen, die m. E. unter keinen Umständen als $\mathrm{gW}$ gelten können.

Zunächst aus Büchmann (2002):

(34) Entenhausen

(35) Quisling

(36) Wandervogel

(37) Blaustrumpf

danach einige Beispiele aus Bramsen (2001):

(38) katharsis

(39) Den røde baron ("Der rote Baron") 
(40) Magna charta

(41) Det tredje rige ("Das Dritte Reich")

Diese Lexeme können kaum jemals zitiert werden, was doch ein Kriterium derselben Wörterbücher ist. Zumindest handelt es sich nicht um ein Zitat in dem Sinne, den ich für diese Kategorie als den einzig zweckmäßigen halte, nämlich als intendierte enzyklopädische Referenz. Der einzige Grund, warum diese und viele andere - Lexeme ins Wörterbuch aufgenommen wurden, ist wohl, dass sie eine Geschichte haben, die erzählt werden kann. Dadurch unterscheiden sie sich aber nicht grundsätzlich von anderen Lexemen, die auch in der Regel eine - wenn auch oft weniger transparente - spannende Etymologie haben. Um gängige Zitate im pragmatischen Sinn handelt es sich $\mathrm{m}$. E. jedoch nicht.

Noch inadäquater ist der vor allem in Bramsen (2001) angewandte Griff, ein Phrasem als gW zu präsentieren, wenn er zufällig bei einem willkürlichen Autor gefunden wurde (s. a. o.) So wird z. B.:

(42) Aldrig i livet ("nie im Leben")

unter Gustaf af Geijerstam (1858-1909) aufgeführt, weil dieser ein Drama mit diesem Titel geschrieben hat. Dabei ist das Phrasem älter als das Stück, und es besteht keine Zitier-Beziehung zwischen dem Phrasem und dem Drama. Schlimmer noch wird es bei:

(43) bringe noget frem i lyset ("etw ins Licht bringen")

das ein jahrhundertaltes Idiom ist, was Bramsen auch selber anführt. Nichstdestoweniger steht das Idiom als gW unter dem modernen Schriftsteller Christian Kampmann (1939-1988) aufgeführt, als würde man diesen Autor zitieren, wenn man das "gW" benutzt. In Wirklichkeit handelt es sich aber um einen für diesen Zusammenhang völlig irrelevanten Idiom-Fund des Lexikografen, den er praktisch überall hätte machen können. Auch ältere Idiome wie:

(44) træde i spinaten ("in den Spinat treten") - (etwa: ins Fettnäpfchen treten)

(45) trække på skuldrene (mit den Achseln zucken)

(46) være på stikkerne - (etwa: auf Zack sein)

gelten bei Bramsen (2001) als gW, weil sie zufällig in den Romanen moderner Schriftsteller wie Ib Michael und Peter Høeg gefunden wurden. Auf diese Weise wird eigentlich der gesamte Wortschatz zu geflügelten Worten, sobald er von einem bekannten Autor verwendet worden ist, was $\mathrm{m}$. E. eine eigenartige Umkehrung des Problems ist.

Die hier angeführten Beispiele aus den beiden Neuerscheinungen sind übrigens keineswegs exklusiv, im Gegenteil sind sie für eine ganze Gattung beispielhaft, die prominente Vertreter wie Scholze-Stubenrecht et al. (1993) und Vogel- 
Jørgensen/Zerlang (1990) mit einschließt. Was hier besprochen wurde, ist also ein generelles Problem der gW-Lexikografie und -Beschreibung.

\section{Was also ist des Pudels Kern?}

Wie oben dargestellt wurde, wird der Begriff „gW“ auf außerordentlich heterogene Weise verwendet. Zunächst ist der unterschiedliche Gebrauch von Laien zu erwähnen, der vielleicht weniger überrascht. Doch auch unter Linguisten sind große Schwankungen zu verzeichnen, dies zeigen sowohl verschiedene Lexikondefinitionen als auch die Einführungen der gW-Spezialwörterbücher. Die Frage ist aber, ob dies für die gW-Forschung das größte Problem darstellt.

Der Inhalt der gängigen gW-Lexika zeigt nämlich, dass der tradiertegWBegriff keineswegs befriedigend sein kann. Denn wenn die Definitionen dazu führen, dass $\mathrm{gW}$ all das sind, das eine irgendwie interessante Geschichte hat, dann trägt der Begriff schon seine eigene Negation in sich. Und zwar einmal weil es ziemlich zufällig ist, ob die Etymologie eines Wortes oder eines Phrasems bekannt ist - und ob eine Erklärung für sicher gehalten wird oder nicht. Dass dies nicht das tragende Kriterium sein kann, zeigt auch die Tatsache, dass ja keineswegs alle Einträge der etymologischen Wörterbücher in die gW-Wörterbücher aufgenommen werden. Hinzu kommt aber, dass eine solche Kategorie linguistisch kaum zu legitimieren ist: sie lebt allein von ihrer lexikografischen Existenz.

Kern der gW ist m. E. der Zitatcharakter, was eine pragmatische Funktion ist. Dagegen ist das zufällige Vorhandensein einer etymologisch-kulturgeschichtlichen Erklärungsmöglichkeit weder eine Funktion noch pragmatisch. Deswegen bilden die Zitat-Einheiten auch in fast allen Fällen immerhin den Hauptstock der Lemmata der $\mathrm{gW}$-Wörterbücher. Leider werden aber, weil sie wohl mit dem Konzept der rörigschen "Sprichwörtlichen Redensarten" (vgl. Rörig 1991) vermischt werden, eine große Menge an Wörtern und Phrasemen mit kodifiziert, die dem Zitatcharakter völlig entbehren oder nicht lexikalisiert sind.

Vorgeschlagen wird somit, den Begriff $\mathrm{gW}$ wissenschaftlich wesentlich einzuengen, wie auch in vielen Fällen intendiert, doch selten praktiziert, und zwar auf die folgenden Merkmale:

- Pragmatische Zitathaftigkeit (intendierte Intertextualität)

- Lexikalisiertheit

Es ist dies eine minimierende gW-Konzeption. Eine solche Konzeption wird zwar nicht unbedingt die Wörterbuchbenutzer zufriedener stellen, andererseits glaube ich, dass sie in höherem Maße der Intuition von Wissenschaftlern und Laien entspricht. Somit werden die $\mathrm{gW}$ zu einer Kategorie, die sich sozusagen 
zwischen den Aphorismen und den (Phraseo)lexemen befindet. Und zwar eine Kategorie, die eine ganz eigene pragmatische Funktion hat, nämlich die des wiederholten Zitiert-Werdens, im Sinne intendierter Bezugnahme auf außersprachliche Tatsachen - (pragmatische) Intertextualität also. Geflügelte Worte sind eine genuin pragmatische, keine philologische Kategorie. Dieses Verständnis sollte sowohl für den theoretischen als auch lexikografischen Umgang mit $\mathrm{gW}$ Folgen haben.

Geflügelte Worte sind - wie jede andere Kategorie der Sprache - selbstverständlich wissenschaftlich beschreibbar (vgl. Abschn. 1). Hier wurde der Versuch getan, eine Revision oder zumindest eine Präzisierung des Begriffs vorzunehmen, die sicherlich dem einen oder anderen Gegner haben wird, der den Vorschlag zu radikal findet. Der Lexikograf sollte aber nicht verzweifeln: Selbst bei einer derart engen wissenschaftlichen Konzeption wird es ihm nicht an Stoff mangeln. Die geflügelten Worte gehören - als wissenschaftlich durchaus vollwertige Objekte - nicht ins sprachliche Raritätenkabinett. Im Gegenteil: Sie sollten allmählich aus demselben herausgeholt werden.

\section{Literatur}

Becker-Christensen, Christian et al. 1999: Nudansk ordbog med etymologi. Kopenhagen: Politiken.

Bergenholtz, Henning/Mogensen, Jens Erik 1993: Wörterbuchkritik in Dänemark. In Lexicographica 9, S. 8-35.

Bramsen, Joachim Bo 2001: Bevingede Ord \& Aforismer. En citatordbog. Kopenhagen: Politiken.

Büchmann, Georg/Robert-Tornow, Walter/Weidling, Konrad/Ippel, Eduard/Krieger, Bogdan/Urban, Eberhard 2001: Der neue Büchmann. Geflügelte Worte. München: Bassermann.

Burger, Harald 20032: Phraseologie. Eine Einführung am Beispiel des Deutschen. Berlin: Erich Schmidt Verlag.

Bußmann, Hadumod 1989: Lexikon der Sprachwissenschaft. Stuttgart: Kröner.

Farø, Ken 2003: Det ligger lige på tungen. Den danske fraseologi. In Mål + Mcele 3/ 2003,19-27.

Fleischer, Wolfgang 1982: Phraseologie der deutschen Gegenwartssprache. Leipzig: VEB Bibliographisches Institut.

Gerr, Elke 2001: Langenscheidt 4000 Sprichwörter und Zitate. Berlin etc.: Langenscheidt.

Glück, Helmut (Hg.) 2000: Metzler Lexikon Sprache. Stuttgart: Metzler.

Google (Internet-Suchmaschine: www.google.com) 
Klosa, Anette/Kunkel-Razum, Kathrin/Scholze-Stubenrecht, Werner/Wermke, Matthias et al. (2001): Duden Deutsches Universalwörterbuch. Mannheim: Dudenverlag.

Rörig, Lutz 1991: Das große deutsche Lexikon der sprichwörtlichen Redensarten. Freiburg im Breisgau: Herder.

Scholze-Stubenrecht, Werner et al. 1993: DUDEN Zitate und Aussprüche. Mannheim etc.: Dudenverlag.

Vogel-Jørgensen, T./Zerlang, Poul 1998: Bevingede ord. Kopenhagen: Gad. 